\title{
VITAGENE REGULATION AS A NEW STRATEGY TO FIGHT STRESSES IN POULTRY PRODUCTION
}

\section{M.A. GRIGORIEVA1, O.A. VELICHKO', S.V. SHABALDIN1, V.I. FISININ², P.F. SURAI $3,4,5,6$}

\author{
${ }^{1}$ AO Prodo Tumen Broiler, s. Kaskara, Tumen Region, Tyumen Province, 625512 Russia, e-mail M.GRIGORE- \\ VA@tumen.prodo.ru; \\ ${ }^{2}$ Federal Scientific Center All-Russian Research and Technological Poultry Institute RAS, Federal Agency of Scientific \\ Organizations, 10, ul. Ptitsegradskaya, Sergiev Posad, Moscow Province, 141315 Russia, e-mail fisinin@land.ru; \\ ${ }^{3}$ Trakia University, Stara Zagora, 6000, Bulgaria; \\ ${ }^{4}$ Szent Istvan University, Godolo, H-2103, Hungary; \\ ${ }^{5}$ K.I. Skryabin Moscow State Academy of Veterinary Medicine and Biotechnology, 23, ul. Akademika K.I. Skryabina, \\ Moscow, 109472 Russia; \\ ${ }^{6}$ Feed-Food Ltd, 53 Dongola Road, Ayr, KA7 3BN, Scotland, UK, e-mail psurai@feedfood.co.uk (corresponding \\ author)
}

ORCID:

Fisinin V.I. orcid.org/0000-0003-0081-6336

Surai P.F. orcid.org/0000-0002-5012-8681

The authors declare no conflict of interests

Acknowledgements:

Peter F. Surai is supported by megagrant from the Government of the Russian Federation (Contract № 14.W03.31.0013) Received May 5, 2017

\section{Abstract}

Commercial poultry production is associated with various stresses leading to decrease of productive and reproductive performance of growing chickens, parent birds and layers. In commercial poultry/animal production, there are four major types of stress: technological, environmental, nutritional and internal stresses. A growing body of evidence indicates that most of the stresses in poultry production at the cellular level are associated with oxidative stress due to excess of free radical production or inadequate antioxidant protection. The vitagene concept of fighting stresses emerged as a new direction in a nutritional research and it was successfully transferred from medical sciences to agricultural sciences, including poultry and pig production. In fact, vitagene regulation by nutritional means appeared as a new approach to realise a full potential of the body for adaptation to stress conditions in poultry/animal production. Therefore, the aim of the study was to test if supplementation of the vitagene-regulating antioxidant mixture (Magic Antistress Mix/PerforMax) with drinking water can improve broiler performance in stressful conditions of commercial chicken production. The experiment was conducted at the poultry farm AO «Prodo Tumenskiy Broiler» (Tumen region, Russia) in a special poultry house designed and equipped for conducting experimental trials and measuring growth parameters weekly. Twelve thousand and four hundred newly hatched Arbor Acres chicks were divided into two equal groups with four replicates in each group and placed in poultry house. The results confirmed the idea that using vitagene-regulating mixture with drinking water can improve chicken performance, including improvement in FCR (1.50 vs 1.56 in control) and vaccination efficacy, as shown by increased vaccination index by $40 \%$ ).

Keywords: chicken, vitagenes, stress, nutrition

Commercial poultry production is associated with various stresses leading to decrease of productive and reproductive performance of growing chickens, parent birds and layers. Indeed, domestication and genetic selection based on rapid growth rates, improved feed conversion, and heavier body weight (BW) of broilers has made domestic birds, including broilers and turkeys, particularly susceptible to oxidative stress [1]. From a physiological point of view, stress is related to a deviation from optimal internal and external conditions. Under stressful conditions, the hypothalamic-pituitary-adrenal axis, the autonomic nervous system and the immune system are responsible for re-establishing homeostasis. Therefore, a cascade of regulatory mechanisms is involved, resulting in a mobilisation of energy and a shift in metabolism, with detrimental effects on growth 
performance and feed efficiency [2]. In modern commercial poultry production, oxidative stress-related nutritional metabolic diseases (e.g. encephalomalacia, exudative diathesis, muscular dystrophy, etc.) have practically disappeared [3, 4], however, various disorders in the antioxidant defence system still cause substantial problems. For example, the amount of a particular nutrient in the diet may be insufficient to meet requirements, the diet may contain substances that inactivate the nutrient or inhibit its absorption/utilisation, or metabolism may be upset by the interaction of dietary and environmental factors causing oxidative stress [5].

A growing body of evidence indicates that most of the stresses in poultry production at the cellular level are associated with oxidative stress due to excess of free radical production or inadequate antioxidant protection [3, 4]. Therefore, dietary antioxidants are considered to be the main protective means to deal with various stresses in poultry production [3, 4, 6-8]. Recently, a concept of the cellular antioxidant defence has been revised with a special attention paid to cell signalling. Indeed, in animals, redox signalling pathways use reactive oxygen species (ROS) to transfer signals from different sources to the nucleus to regulate a number of various functions including growth, differentiation, proliferation and apoptosis.

The vitagene concept of fighting stresses emerged as a new direction in a nutritional research and medical sciences. In accordance with Calabrese et al. [9] the term vitagenes refers to a group of genes that are strictly involved in preserving cellular homeostasis during stress conditions and playing an essential regulatory role in cell and whole organism adaptation to various stresses. The vitagene family includes heat shock proteins (HSPs; Heme oxygenase-1 and HSP70), the thioredoxins (Trx)/thioredoxin reductase (TrxR) system, sirtuins [9] and superoxide dismutases (SOD) [10]. The vitagene concept has been applied to various human diseases, involving neurodegenerative disorders [11], neuroprotection [12], schizophrenia [13], vascular dementia [14], autism spectrum disorders [15], aging and longevity [9, 16-18], dermatology [19], free radical-related diseases [20], osteoporosis and Alzheimer pathology [21]. The vitagene concept was successfully transferred from medical sciences to agricultural sciences, including poultry and pig production [22-28] and was reviewed in previous issue of this journal 5 years ago [23]. Upregulating vitagenes, for-example, via the Nrf2/ARE system, and improving adaptive ability of animals to stress it is possible to decrease negative consequences of various stresses in poultry and farm animal production. Indeed, vitagene regulation by nutritional means $[10,23,27$, 28] appeared as a new approach to realise a full potential of the body for adaptation to stress conditions in poultry/animal production. Indeed, modulation of endogenous cellular defence mechanisms may be an innovative approach to deal with commercially relevant stresses in poultry/animal production.

Therefore, when testing whether supplementation of the vitageneregulating antioxidant mixture (Magic Antistress Mix/PerforMax) can improve broiler performance in stressful conditions of commercial chicken production, in this well organised and controlled experiment, conducted in accordance with requirements set in many countries with developed poultry production, it was clearly shown that Magic Antistress Mix/PerforMax supplied with drinking water to Arbore Acres broilers, decreased negative impact of commercial stresses. This led to improvement of feed conversion ratio (FCR) and vaccination index (VI).

The aim of the study was to test if our previously developed vitageneregulating antioxidant mixture (Magic Antistress Mix/PerforMax) can be used to improve liveability and productivity of broilers in commercial conditions.

Techniques. The experiment was conducted at the poultry farm AO Pro- 
do Tyumen Broiler (Tumen region, Russia) in a special poultry house designed and equipped for conducting experimental trials and measuring growth parameters weekly. Twelve thousand and four hundred newly hatched Arbor Acres chicks were divided into two equal groups with four replicates in each group and placed in a poultry house. Temperature, light and other parameters of chicken growth were maintained in accordance with recommendations of Arbor Acres accepted at the poultry farm. The diet was balances in all major nutrients in accordance with the same Arbor Acres recommendations accepted at the poultry farm. In additional to the balances died the experimental birds were supplemented with Magic Antistress Mix/PerforMax with drinking water at a dose of $1 \mathrm{mg} / \mathrm{l}$ in stressful period of chicken growth in accordance with recommendations of the preparation developer (Feed-Food Ltd., UK). The preparation composition was shown earlier to contain vitagene-regulating mixture of vitamins, minerals, amino acids, carnitine, betaine, organic acids, etc. [35].

Chickens were grown up to 35 days and information was collected weekly. Body weight and daily gain (g), mortality (\%), daily feed consumption (g) and FCR were assessed in three growth periods (15-21 days, 22-28 days and 2935 days) and for whole growth period (1-35 days). To compare broiler results from different flocks and different regions, the European Broiler Index (EBI) or European Production Efficiency Factor (EPEF) is used (this factor standardises technical results, taking into account feed conversion, mortality and daily gain):

$\mathrm{EPEF}=($ Average daily gain, $\mathrm{g} \times$ survival rate, $\%) /(\mathrm{FCR} \times 10)$.

At the end of the experiment intestinal weight was assessed and internal organ examination was conducted to check nephritis, enteritis and intestinal atony.

1. Effect of usage with drinking water of vitagene-stimulating additive Magic Antistress Mix/PerforMax on growth and development of Arbor Acres broilers $(M \pm S E M$, the experiment, AO Prodo, Tyumen broiler, Tyumen region)

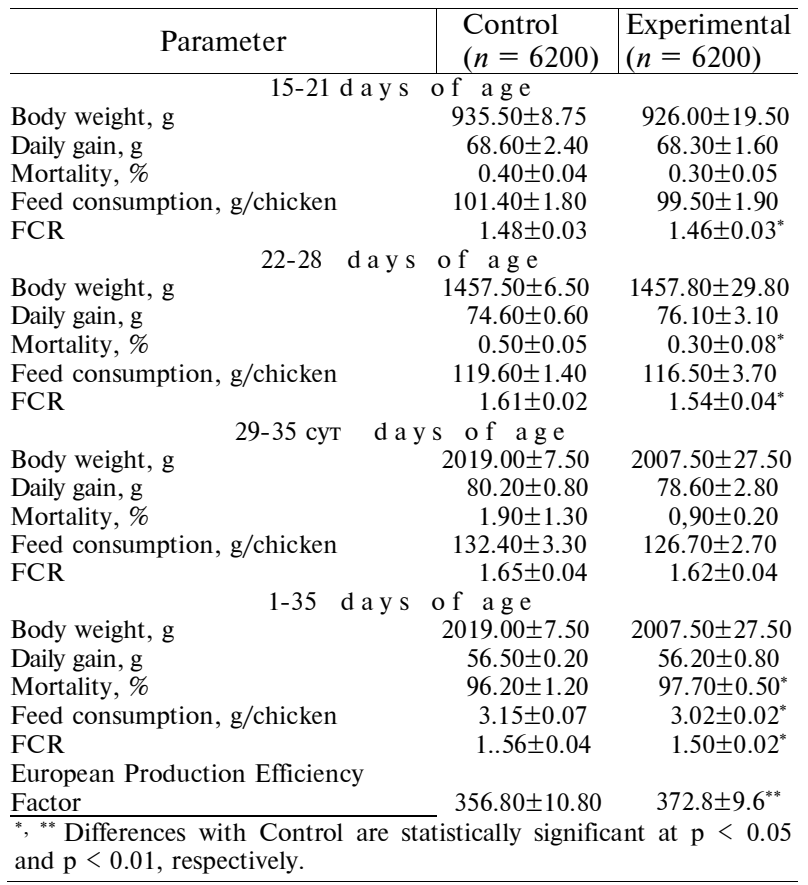

Efficacy of vaccination against infectious bursitis was assessed by ELISA in accordance with recommendations of test system producer (BioTek Instruments, Inc., USA). Vaccination Index (VI) was calculated as a ratio of average antibody titre by ELISA to variation coefficient $(C v, \%)$.

During statistical analysis, average parameters $(M)$ and the standard errors $( \pm$ SEM) were determined. Significance of differences with Control was assessed by $t$-test by using ANOVA, differences were considered to be significant at $\mathrm{p}<0.05$.

Results. Chicken viability (Table 1) in both groups was within standards for the poultry farm. However, the mortality for 35 days in the control group (3.8\%) was numerically higher in comparison to the control group. In commercial conditions such a difference could be significant for a meat producer. Usage of Magic Antistress Mix/PerforMax with drinking water was associated with 
decrease number of enteritis, nephritis and intestinal atony as well as pododermatitis (data not shown). For the first two weeks of the experimental period data in the control and experimental groups were not different (data not shown). Later (see Table 1) there was an improvement in FCR in the experimental group in comparison to the control group resulting in decreased feed consumption per week. There was no difference in body weight and daily gain in the control and experimental groups. Therefore, the main advantage of usage of vitageneactivating composition for growing broilers was associated with improvement of FCR, which in the experimental group (1.50) was significantly better than in the control group (1.56).

EPEF is used to compare results of broiler growth in different areas and flocks. Taking into account all data of the chicken growth and development for 5 weeks EPEF in the control group was shown to be 356.8, while in the experimental group it was 372.8 , being comparable with an efficacy of broiler production at the best farms in Russia and abroad.

It is important to mention that in experimental chickens at day 35 intestinal weight was higher (by $5 \%$ ) in comparison to the control chickens (Fig. 1, A).
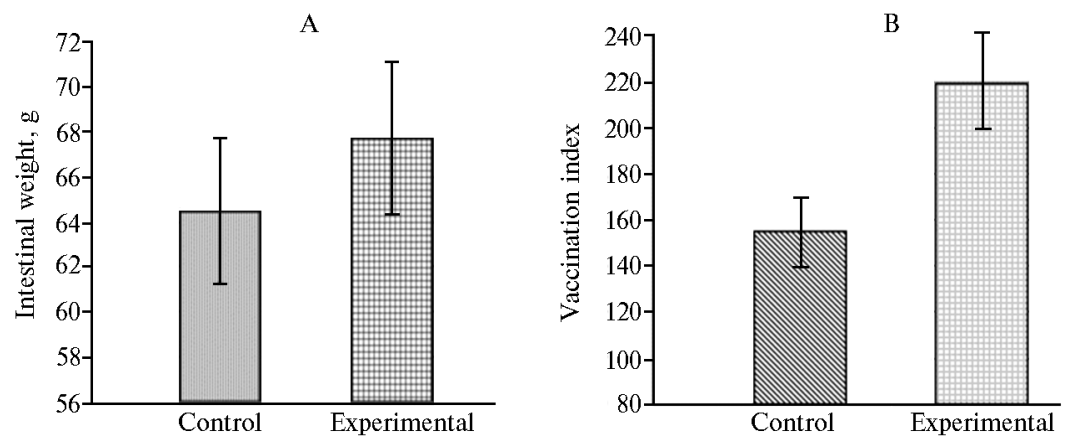

Fig. 1. Intestinal weight (A) and vaccination index (B) in 35-day old Arbor Acres broilers as a result of providing with drinking water the vitagene-stimulating additive Magic Antistress Mix/PerforMax (the experiment, AO Prodo Tyumen broiler, Tyumen region, Russia).

Vaccination efficacy against infectious bursal disease assessed by ELISA methodology (BioTeck) at day 35 includes antibody titres, $C v$ expressed in \% and a vaccination index calculated as a ratio of average antibody titre to $C v$. Our data indicates that average antibody titre in 35 days old chickens was 4500 in the control group and 5258 in the experimental group. It is interesting that $C v$ in the experimental group (24\%) was lower than that in the control group (29\%). As a result, the vaccination index in the experimental group was significantly higher than that in the control group (see Fig. 1, B).

2. Economics (in roubles) of Arbor Acres broiler meat production with usage with drinking water of vitagene-stimulating additive Magic Antistress Mix/PerforMax (the experiment, AO Prodo Tyumen broiler, Tyumen region, Russia)

\begin{tabular}{|c|c|c|c|}
\hline Economical parameters & Control & Experimental & Difference \\
\hline \multicolumn{4}{|l|}{ Cost of $1 \mathrm{~kg}$ meat production : } \\
\hline salary with taxes & 2.09 & 2.09 & 0.00 \\
\hline Feed & 33.74 & 32.45 & -1.30 \\
\hline Veterinary & 1.99 & 2.19 & 0.20 \\
\hline Other & 8.21 & 8.13 & -0.08 \\
\hline Cost of day old chick per $1 \mathrm{~kg}$ meat production & 6.67 & 6.61 & -0.07 \\
\hline Cost of $1 \mathrm{~kg}$ live weight & 52.71 & 51.47 & -1.24 \\
\hline Cost $1 \mathrm{~kg}$ of meat production & 70.24 & 68.59 & -1.66 \\
\hline Cost of slaughtering and sale per $1 \mathrm{~kg}$ meat & 19.61 & 19.61 & 0.00 \\
\hline Average price of meat sales per $1 \mathrm{~kg}$ & 120.69 & 120.69 & 0.00 \\
\hline Income per $1 \mathrm{~kg}$ meat produced & 30.84 & 32.49 & 1.66 \\
\hline
\end{tabular}


As a result of FCR improvement due to water application of the antistress composition economics of meat production was improved (Table 2). Indeed, due to the PerforMax application an additional income per $\mathrm{kg}$ of meat production comprises 1.66 roubles.

In discussion of the data it is necessary to mention that in poultry production, there are four major types of stress [25-27], including technological, environmental, nutritional and internal stresses [28-33] (Fig. 2).

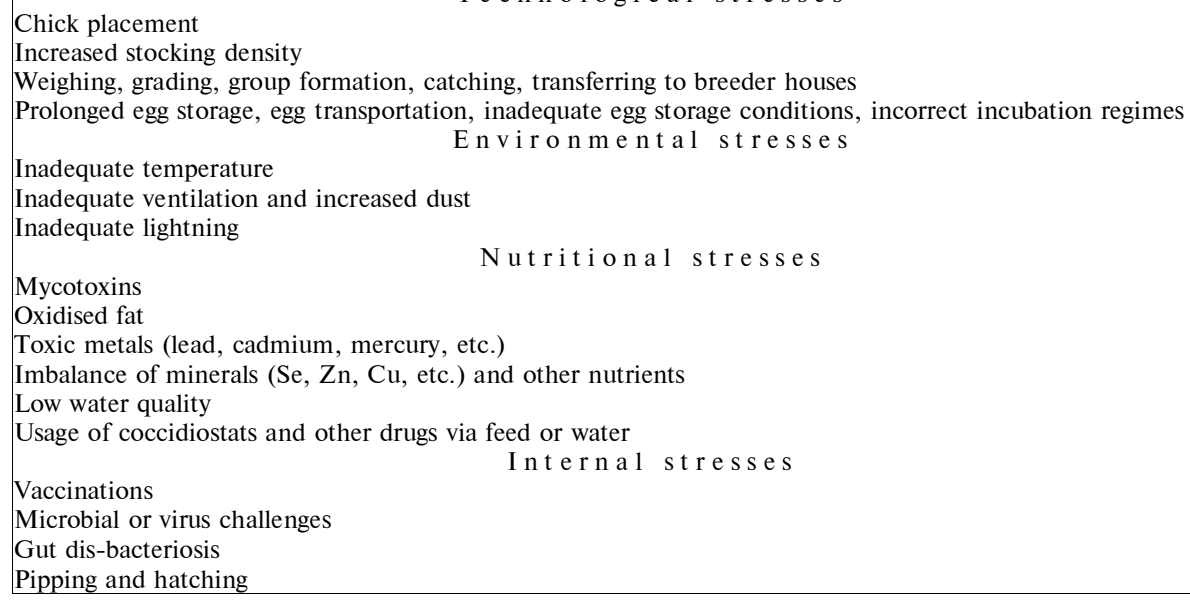

Fig. 2. Stresses in poultry production, adapted from [30].

It has been suggested that in broiler production stress of chicken placement is one of the most important stresses. Indeed, chick viability is an important factor in determining profitability. In fact, from fertilisation to placement at the broiler farm, factors such as egg quality, egg storage conditions, incubation conditions and post-hatch environment will all affect chick quality [34]. It has been proven that the first 24 hours of the chick's life are the most important [34-37]. It is believed that a chick should have an access to the feed and water as soon as possible after hatching to stimulate the development of the digestive and immune systems. It is well appreciated that time between chick hatch and placement is stressful due to dehydration and yolk sac reserve depletion. Indeed, when putting together hatching time inside the hatcher, time of chick processing and transportation, and finally, placement at the farm, it could take up to $36-48 \mathrm{~h}$ before a newly hatched chick has access to feed and water and during this time body weight decreases quickly [38]. It has been shown that in the hatching chick the most dramatic changes in the small intestine occur within the first $24 \mathrm{~h}$ posthatch [39]. In fact, there is an inverse relationship between duration of posthatching holding time and subsequent chick performance [34-37, 40, 41]. Therefore, immediate access to feed and water help achieving an increased body weight of the growing chick at 3 weeks of age [42] or at market age of broilers [43]. It should be also mentioned that there is the hatch window (24-36 hours) or the spread between late and early hatchers which depends on the homogeneity/heterogeneity of the incubating eggs which is dependent on breeder age [36, 37]. A spread in the hatching period will increase the numbers of chicks sitting extra hours in stressful conditions of the hatcher without food or water. Furthermore, any delay in accessing food [44, 45] and/or water intake after hatching as well as hatchery treatments such as vaccination, sexing and transport to the farm can result in additional stress [46]. Indeed, extended time in the hatcher $(36 \mathrm{~h})$ was associated with decreasing antioxidant defences indicative by decreased vitamin E and coenzyme Q concentrations in chicken tissues [47]. 
Given the relatively high temperature and humidity in the hatcher, it is possible to make the argument that the chick may be under chronic oxidative stress during this holding time [23, 47]. Therefore, antioxidant protection at hatching time is considered to be an important determinant of chick viability during first posthatch days [3, 48-50]. During chick embryo development there is an antioxidant/prooxidant balance in the tissues which supports normal embryonic development and post-hatch chick viability [48-51]. It has been suggested that an accumulation of the natural antioxidants like vitamins A, E and carotenoids as well as an increase in GSH-Px activity in the embryonic liver may have an adaptive significance, evolving to protect unsaturated lipids against peroxidation during the stress imposed by hatching [3, 49, 50].

Postnatal nutritional exposures are considered to be critical for the developmental maturation of many organ systems and optimal physiological functions. There is a growing body of evidence indicating that environmental exposures including nutritional exposures during these critical and sensitive periods of life can cause permanent changes in many physiological processes, which is known as "programing" [52]. Our previous investigations indicate that low quality neonatal nutrition resulted in long-term impairment in the capacity to assimilate dietary antioxidants [53]. It seems likely that early programming associated with epigenetic mechanisms plays a key role in chicken growth and development and at time of chicken placement. Furthermore, scientific evidence is accumulating that the programming effects of conditions during early development can be transmitted to the offspring [54]. Therefore, transgenerational effects of stress are potentially mediated via modulation of the hypothalamic-pituitary-adrenal axis as well as epigenetic mechanisms causing heritable changes in gene expression and it was suggested that early experiences may shape phenotypes of chickens in a long-term way [55]. In addition to the aforementioned stresses chicks are exposed to such stresses as hatching without maternal contact, transportation and social isolation. Indeed, the early life social isolation stress resulted in a dampened corticosterone response to restraint stress in affected birds and in their male offspring. Furthermore, stress-specific genes, such as early growth response 1 and corticotropin-releasing hormone receptor were upregulated immediately after restraint stress [55].

Research data is accumulating to support the hypothesis that the vitamin E status of chickens and turkey poults and chickens may be inadequate during the first weeks after hatching [56]. A variety of approaches aimed at improving the vitamin E status of turkey poults have, in fact, been investigated including dietary supplementation of the poults with high levels of $\alpha$-tocopherol [3, 57], bile salts [58] and fat [59], as well as vitamin E injection [60] and alterations in provision of n-6 and n-3 polyunsaturated fatty acids [60]. When $d-\alpha$-tocopherol was added in the drinking water, there was an increase of $\alpha$-tocopherol in tissues and a decreased susceptibility of red blood cells to hemolysis [61]. Moreover, dayold chickens were treated per os with $3.25 \mathrm{mg}$ vitamin E/bird per day, via the drinking water, for two weeks. The vitamin E content of both the liver and the blood plasma was significantly higher in the treated chickens than in the untreated controls [62]. It seems likely, that provision of vitamin $\mathrm{E}$ and other fatsoluble vitamins $\left(A\right.$, and $\left.\mathrm{D}_{3}\right)$ with water at time of chicken placement can solve the problem of their low availability for newly hatched chicks [23]. Such a supplementation helps chickens overcome stress of placement and has positive effects on chicken growth and development.

When chicks are placed in winter while outside temperature is quite low there is always a temptation to decrease ventilation to keep energy usage to the minimum. However, it is very important to provide good quality, warm, fresh air 
that is rich in oxygen for the recently hatched chicks. Indeed, the chick's trachea is very often irritated from being boxed and shipped in the chick trays, often for many hours. Furthermore, chicks can be exposed to formaldehyde gas and contaminated air during hatch $[23,50]$. Excessive amounts of irritants such as carbon dioxide and ammonia can cause depression, dehydration, emaciation as well as various problems with the respiratory system of the chick [23, 50]. The increased lipid peroxidation and reduced activities of antioxidant enzymes in healthy chickens reared under unfavourable microclimatic conditions such as higher air temperature and humidity, higher ammonia concentrations, and lower light intensity were indicative about an induced oxidative stress [63]. It should also be mentioned that poor ventilation is often associated with toxic carbon monoxide accumulation. Toxicity causes irreversible physiological and biochemical changes that cannot be corrected with successive additional ventilation [25, 26]. Therefore, to deal with oxidative stress at chicken placement, there are several important options. They include: a) electrolyte supplementation via drinking system to increase water consumption by chicks and keep optimal electrolyte balance in the body [23]; b) fat-soluble vitamin supplementation via drinking water to overcome low efficacy of vitamin assimilation from the diet $[3,23]$; c) other protective nutrient supplementation (ascorbic acid, Se, carnitine, betaine, lysine, methionine, etc.) with water to decrease oxidative stress related to chick placement and gut adaptation to a new type of feed [23, 28]. Improved antioxidant defences during first days of postnatal life are suggested to help immune system development in this critical period of time $[65,66]$.

As it was shown in our study, supplementation of the vitagene-regulating composition with drinking water helps chicken to maintain optimal FCR which was significantly improved in comparison to the control chickens. Therefore, it could well be that by decreasing placement stress it is possible to give chickens a better start which later will be translated into better gut development and health. In fact a trend indicating increased intestinal weight in the experimental chicks found in our study could be one of those factors reflecting the aforementioned feature. Our previous idea about antioxidant-prooxidant balance in the gut [67, 68] recently has been updated in relation to chicken placement [51]. Indeed, antioxidant protection in chicken gut is based on natural antioxidants, including vitamin E and carotenoids, and enzymatic systems [69], including SOD, an important part of the vitagene network [10]. Therefore, by using vitagenepromoting composition with drinking water it is possible to improve antioxidant defences of the gut and this could contribute to a better gut integrity translated into a better FCR.

The second possibility of better FCR is an improvement of immunocompetence. Indeed, immune system is a crucial factor determining not only chicken health but also chicken production efficacy. In fact, immune system is quite expensive to run and only optimal immune response gives a reliable protection against pathogens with minimal expenses for the body. Our data indicates that index of vaccination in the experimental chickens was increased and this could be due to immunomodulating properties of the vitagene-regulating mixture used in this experiment. Our previous studies clearly showed that upregulation of vitagenes is an important approach for high immunocompetence in commercial poultry production and PerforMax was shown to decrease vaccination stress [23, 66]. It has been proposed that communication between various immune cells is a key for high immunocompetence [3, 4]. Indeed, immune system includes trillions of lymphocytes and billions of phagocytes and all those cells have to communicate with each other and other types of immune cells to make a right decision on the strategy of immune defence. It seems likely that receptors on the 
surfaces of immune cells are responsible for such a communication and the receptors can be damaged by free radicals in stress conditions. Furthermore, production of communicating molecules such as cytokines and eicosanoids can also be affected by oxidative stress. Therefore, by upregulating vitagenes responsible for synthesis of such protective molecules as heat shock proteins, thioredoxin and thioredoxin reductase, sirtuins and superoxide dismutase it is possible to provide a protection for receptors and maintain high immunocompetence in stress conditions.

Our previous studies indicated positive effects of the vitagene-regulating mixture (Magic Antistress Mix/PerforMax) on growing broilers, including improvement FCR [71, 72] and improved immunocompetence [70]. In this study previous results were confirmed in conditions providing growth, development and FCR comparable with the best examples of chicken production in Russia and abroad generating additional income.

Much attention has recently been paid to mycotoxins as the main nutritional stresses in poultry and animal production [73-76]. Oxidative stress caused by mycotoxins is the main molecular mechanism of their action [77-79]. This is true first of all for ochratoxin [80-83], T-2 toxin [84-86], DON [87, 89], fumonisins [90-92] and aflatoxins [93, 94]. It is very difficult to prevent feed contamination by mycotoxins. That is why various adsorbents are used to decrease negative effects of mycotoxins on animal/poultry with varied success [95-98]. However, absorptive capacity for mycotoxins in different adsorbents is greatly varied and, therefore, feed adsorbents are not able to solve mycotoxin problem in animal/poultry industry. It seems likely that key strategy in fighting the mycotoxin problem includes usage of various antioxidant compositions in the diet to support functions of the liver, a place where detoxification of most mycotoxins takes place as well as to support intestine where microbiota is involved in DON detoxification [78, 99]. Indeed, successfully tasted in our experiment the vitagene-stimulating composition of wide spectrum of action may become a very important instrument in the hands of poultry technologists/nutritionists [22, 23].

The importance and efficacy of the vitagene-regulating composition for rearing birds and adult egg type breeders (Hy-Line) have been studied at one of the biggest egg producing farms in Russia (Borovskaya poultry farm, Tumen region) and have been recently reviewed [28, 33, 100]. In particular, it was shown that usage of the antistress composition containing vitagene-activating nutrients (PerforMax/Magic Antistress Mix) with drinking water at specific periods of the increased stress can improve breeders' performance. In particular, there was an increase by $2 \%$ of the egg peak production and peak plateau lasted about 50 days longer than that in the control birds. It is interesting to note that egg production in the control hens (260.8 eggs) was higher than the target one for the line in Russia (253.4 eggs), and in the experimental group this parameter increased by 6 eggs. Furthermore, improved egg production was associated with increased weight of the oviduct in the experimental layers. It is also important to mention that FCR (feed per 10 eggs) was also improved by usage of the antistress composition and was better than the target one for the line. Notably, shell strength at age 26, 36 and 56 weeks was improved in the experimental group by 2.8, 5.6 and $5.6 \%$, respectively. The most interesting finding was related to a significant increase of the carotenoid level in the egg yolk of experimental birds. Since carotenoids were not supplied with the antistress composition, this increase could be due to improved absorption of nutrients resulting from antistress composition usage. This can also explain improved FCR in the experimental birds. Vitamin A level in the egg yolk from the experimental layers was also increased probably reflecting its transfer from the antistress composition. In particular, antistress 
composition usage was associated with improved fertility at weeks 16, 40, 48 and 56 by $2.5 ; 2.7 ; 2.8$ and $3.7 \%$, respectively. In the same experimental group the hatch of condition chicks improved at weeks $26,32,40,48$ and 56 by $3.6 ; 2.1$; $3.4 ; 4.9$ and $4.3 \%$, respectively $[28,33,100,101]$. Similarly, effects of the antistress composition on the rearing birds were studied [28]. In fact, the usage of the antistress composition positively affected testes development of 15-, 26- and 56 -week old cockerels. The liver of experimental birds was characterised by a significant increase in vitamin A content at various ages [28, 102]. It was clearly shown that in laying type breeders PerforMax increased egg production, improved percentage of eggs suitable for incubation, as well as improved hatchability. This gave a profitability of hatching egg production at $29.3 \%$ in comparison to $19.7 \%$ in the control group [28]. There is also a possibility of increasing hatching egg production in broiler breeders due to PerforMax usage [103]. Furthermore, possible effects of supplying the antistress composition at $1 \mathrm{~g} / \mathrm{l}$ drinking water to Hy-Line breeders on their progeny chicks with specific emphasis to chick uniformity as an important determinant of rearing birds' quality were studied [104]. The obtained data indicates that supplying the antistress composition to breeders was associated with a significant improvement of the uniformity (at day 28) in progeny chicks obtained from breeders of various ages: 26 weeks $(81.3$ vs $67.3 \%)$, 32 weeks ( 85.5 vs $76.8 \%)$, 40 weeks ( 83.2 vs $68.8 \%$ ), 48 weeks (75.5 vs $68 \%$ ) and 56 weeks (73.7 vs $62 \%)$. Therefore, it seems likely that changes in egg composition due to supplying to breeders with important nutrients, including methyl donors (betaine, methionine, vitamin $\mathrm{B}_{12}$, etc.), could have epigenetic effects of the progeny chicks [23, 104, 105]. Furthermore, protective effect sof the product in pig production were also shown [24, 51, 106]. Our preliminary studies also indicate that upregulation of vitagenes by nutritional means is a universal approach for fighting stresses, since usage of vitageneregulating composition in other avian (turkey, duck, goose, quail) and animal species (young calves, lambs, foals, rabbits, fur animals, companion animals, fish, etc.) is quite effective (P. Surai, unpublished).

A study of relationship between vitagenes and gut microbiota can open new chapter in understanding of the role of gut immunity and structural integrity of the intestine in poultry/animal health [107-110]. It seems likely that targeted action on the vitagene expression in the intestine could help find new approaches for increasing poultry/animal resistance to microbial and viral pathogens. However, this question remains to be studied in the future.

Thus, our new concept of fighting stresses is based on an idea that supplying birds with various antioxidants via drinking water could help them deal with stress conditions more effectively. In the aforementioned study it was confirmed that usage of the vitagene-regulation composition supplied with drinking water is an effective means in fighting stresses and improving growing birds' performance, including improvement in FCR and improved vaccination efficacy. The cooperative mechanisms of the vitagene network are considered in detail in recently published comprehensive reviews with a major conclusion indicating an essential regulatory role of the vitagene network in cell and whole organism adaptation to various stresses. Indeed, the products of the vitagenes actively operate in detecting and controlling diverse forms of stress and cell injuries. Furthermore, our analysis of recent data indicates that vitagenes can be regulated by nutritional means. In particular, vitamins A, D, E, C, as well as selenium, carnitine and betaine are shown to affect vitagenes and improve adaptive ability of animals/birds to various stresses. In fact, protective effects of the aforementioned nutrients on vitagenes are most pronounced in stress conditions. Indeed, these results are the first step to go from the development of 
the vitagene concept to designing a commercial product which can help fighting stresses in the commercial conditions of poultry and pig production. However, further work is required to understand molecular mechanisms of the interactions of vitagenes with various signaling pathways and transcription factors in the cell to build an adequate adaptive response to minimize detrimental consequences of commercially-relevant stresses in poultry and pig production. This would give an opportunity to improve our universal antistress composition and develop more specific compositions to deal with liver disorders in poultry associated with a commercial production of eggs and meat. It is likely that the vitagene concept would help solving other problems of modern poultry production, including losses associated with egg shell quality and eggs cracks in layers at the second part of their production.

\section{R E F E R E N C E S}

1. Soleimani A.F., Zulkifli I., O mar A.R., Raha A.R. Physiological responses of 3 chicken breeds to acute heat stress. Poultry Sci., 2011, 90: 1435-1440 (doi: 10.3382/ps.2011-01381).

2. Bureau C., Hennequet-Antier C., Couty M., Guemene D. Gene array analysis of adrenal glands in broiler chickens following ACTH treatment. BMC Genomics, 2009, 10: 430 (doi: 10.3201/eid1508.080772).

3. S u ra i P.F. Natural antioxidants in avian nutrition and reproduction. Nottingham University Press, Nottingham, 2002.

4. S u r a i P.F. Selenium in nutrition and health. Nottingham University Press, Nottingham, 2006.

5. Mezes M., Surai P.F., Salyi G., Speake B.K., Gaal T., Maldjian A. Nutritional metabolic diseases in poultry and disorders of the biological antioxidant defence system. Acta Veterinaria Hungarica, 1997, 45: 349-360.

6. Fe 11 e n berg M.A., S p e is ky H. Antioxidants: Their effects on broiler oxidative stress and its meat oxidative stability. World's Poultry Science Journal, 2006, 62: 53-70.

7. Est e vez M. Oxidative damage to poultry: from farm to fork. Poultry Sci., 2015, 94: 13681378 (doi: 10.3382/ps/pev094).

8. Akbarian A., Michiels J., Degroote J., Majdeddin M., Golian A., De $\mathrm{S} \mathrm{m}$ e $\mathrm{t}$ S. Association between heat stress and oxidative stress in poultry; mitochondrial dysfunction and dietary interventions with phytochemicals. J. Anim. Sci. Biotechnol., 2016, 7: 37 (doi: 10.1186/s40104-016-0097-5).

9. Calabrese V., Guagliano E., Sapienza M., Panebianco M., Calafato S., Puleo E., Pennisi G., Mancuso C., Butterfield D.A., Stella A.G. Redox regulation of cellular stress response in aging and neurodegenerative disorders: role of vitagenes. Neurochem. Res., 2007, 32: 757-773.

10. Surai P.F. Antioxidant systems in poultry biology: superoxide dismutase. Journal of Animal Research and Nutrition, 2015, 1: 1-17.

11. Calabrese V., Boyd-Kimball D., Scapagnini G., Butterfield D.A. Nitric oxide and cellular stress response in brain aging and neurodegenerative disorders: the role of vitagenes. In Vivo, 2004, 18: 245-267.

12. Calabrese V., Cornelius C., Mancuso C., B a rone E., Cal a fato S., B a tes T., Rizzare 11 i E., Kostova A.T. Vitagenes, dietary antioxidants and neuroprotection in neurodegenerative diseases. Frontiers in Bioscience, 2009, 14: 376-397.

13. Calabrese V., Giordano J., Crupi R., Di Paola R., Ruggieri M., Bianchini R., Ontario M.L., Cuzzocrea S., C a labrese E.J. Hormesis, cellular stress response and neuroinflammation in schizophrenia: Early onset versus late onset state. $J$. Neurosci. Res., 2017, 95(5): 1182-1193 (doi: 10.1002/jnr.23967).

14. Calabrese V., Giordano J., Signorile A., Laura Ontario M., Castorina S., De Pasquale C., Eckert G., Calabrese E.J. Major pathogenic mechanisms in vascular dementia: Roles of cellular stress response and hormesis in neuroprotection. $J$. Neurosci. Res., 2016, 94: 1588-1603 (doi: 10.1002/jnr.23925).

15. Calabrese V., Giordano J., Ruggieri M., Berritta D., Trovato A., Ont a ri o M.L., B i a n chini R., Ca labres e E.J. Hormesis, cellular stress response, and redox homeostasis in autism spectrum disorders. J. Neurosci. Res., 2016, 94: 1488-1498 (doi: 10.1002/jnr.23893).

16. Calabrese V., Cornelius C., Cuzzocrea S., Iavicoli I., Rizzarelli E., Calabrese E.J. Hormesis, cellular stress response and vitagenes as critical determinants in aging and longevity. Molecular Aspects of Medicine, 2011, 32: 279-304 (doi: 10.1016/j.mam.2011.10.007).

17. Calabrese V., Cornelius C., Dinkova-Kostova A.T., Iavicoli I., Di Paola R., Koverech A., Cuzzocrea S., Rizzarelli E., Cal abrese E.J. Cellular stress responses, hermetic phytochemicals and vitagenes in aging and longevity. $B B A, 2012$, 1822: 753-783 (doi: 10.1016/j.bbadis.2011.11.002). 
18. Calabrese V., Scapagnini G., Davinelli S., Koverech G., Koverech A., De Pasquale C., Salinaro A.T., Scuto M., Calabrese E.J., Genazzani A.R. Sex hormonal regulation and hormesis in aging and longevity: role of vitagenes. Journal of Cell Communication and Signalling, 2014, 8: 369-384 (doi: 10.1007/s12079-014-0253-7).

19. Calabrese V., Calafato S., Puleo E., Cornelius C., Sapienza M., Morg a nti P., M a n cus o C. Redox regulation of cellular stress response by ferulic acid ethyl ester in human dermal fibroblasts: role of vitagenes. Clinics in Dermatology, 2008, 26: 358-363 (doi: 10.1016/j.clindermatol.2008.01.005).

20. Calabrese V., Cornelius C., Trovato A., Cavallaro M., Mancuso C., Di Rienzo L., Condorelli D., D e Lorenzo A., Calabrese E.J. The hormetic role of dietary antioxidants in free radical-related diseases. Current Pharmaceutical Design, 2010, 16: 877-883.

21. Cornelius C., Koverech G., Crupi R., Di Paola R., Koverech A., Lodato F., Scuto M., S a linaro A.T., Cuzzocrea S., Calabrese E.J., Calabrese V. Osteoporosis and Alzheimer pathology: Role of cellular stress response and hormetic redox signaling in aging and bone remodeling. Frontiers in Pharmacology, 2014, 5: 120 (doi: 10.3389/fphar.2014.00120).

22. F is i n i n V.I., S u r a i P.F. Ptitsa i ptitseprodukty, 2011: 5: 23-26 (in Russ.).

23. S u rai P.F., Fis in in V.I. The modern anti-stress technologies in poultry production: from antioxidants to vitagenes. Sel'skokhozyaistvennaya biologiya [Agricultural Biology], 2012, 4: 3-13 (doi: 10.15389/agrobiology.2012.4.3eng).

24. S u r a i P.F., M e l' $\mathrm{n}$ i c hu k S.D. Svinovodstvo Ukrainy, 2012, 2: 10-15 (in Russ.).

25. S u ra i P.F., Fis i n i n V.I. Vitagenes in poultry production. Part 1. Technological and environmental stresses. World's Poultry Science Journal, 2016, 72, 721-733 (in Russ.).

26. S u rai P.F., F is in in V.I. Vitagenes in poultry production. Part 2. Nutritional and Internal stresses. World's Poultry Science Journal, 2016, 72: 761-772.

27. S u ra i P.F., Fis in in V.I. Vitagenes in poultry production. Part 3. Vitagene concept development. World's Poultry Science Journal, 2016, 72: 793- 804.

28. S hat s k i k h E.V., L a t y p o va E.N., N e s ve t U.G., K o b u r n e e v I.V. Ispol'zovanie antistressovykh preparatov $v$ yaichnom ptitsevodstve [Application of anti-stress compositions in egg commercial production]. Ekaterinburg, 2016 (in Russ.)

29. S c a n e s CG. Biology of stress in poultry with emphasis on glucocorticoids and the heterophil to lymphocyte ratio. Poultry Sci., 2016, 95: 2208-2215 (doi: 10.3382/ps/pew137).

30. G e s s ne r D.K., Ring s e is R., Ed e r K. Potential of plant polyphenols to combat oxidative stress and inflammatory processes in farm animals. J. Anim. Physiol. Anim. Nutr. (Berl.), 2017, 101: 605-628 (doi: 10.1111/jpn. 12579).

31. Habibian M., S a d e ghi G., Ghazi S., Mo e in i M.M. Selenium as a feed supplement for heat-stressed poultry: a review. Biol. Trace Elem. Res., 2015, 165: 183-193 (doi: 10.1007/s12011015-0275-x)

32. S a e ed M., B abazadeh D., N ave ed M., Arain M.A., Has san F.U., Chao S. Reconsidering betaine as a natural anti-heat stress agent in poultry industry: a review. Trop. Anim. Health Prod., 2017, First Online 21 July: 1-10 (doi: 10.1007/s11250-017-1355-z).

33. Shatskih E., Latipova E., Fis in in V., De nev S., Surai P. Molecular mechanisms and new strategies to fight stresses in egg-producing birds. Agric. Sci. Technol., 2015, 7: 3-10.

34. De cuypere E., T o n a K., B rugge m a n V., B a me lis F. The day-old chick: a crucial hinge between breeders and broilers. World's Poultry Science Journal, 2001, 57: 127-138.

35. N o y Y., U n i Z. Early nutrition strategy. World's Poultry Science Journal, 2010, 66: 639-646.

36. Cherian G. Nutrition and metabolism in poultry: role of lipids in early diet. J. Anim. Sci. Biotechnol., 2015, 6(1): 28 (doi: 10.1186/s40104-015-0029-9).

37. Wang Y., Li Y., Willems E., Wille msen H., Franssens L., Koppenol A., Guo X., Tona K., Decuypere E., Buyse J., Everaert N. Spread of hatch and delayed feed access affect post hatch performance of female broiler chicks up to day 5. Animal, 2014, 8(4): 610-617.

38. Noy Y., Skla n D. Different types of early feeding and performance in chicks and poults. The Journal of Applied Poultry Research, 1999, 8: 16-24.

39. G e y ra A., U n i Z., S k la n D. Enterocyte dynamics and mucosal development in the posthatch chick. Poultry Sci., 2001, 80: 776-782.

40. Hage r J.E., B e a n e W.L. Posthatch incubation time and early growth of broiler chickens. Poultry Sci., 1983, 62: 247-254.

41. Pin chas ov Y., N o y Y. Comparison of post-hatch holding time and subsequent early performance of broiler chicks and Turkey poults. British Poultry Science, 1993, 34: 111-120.

42. Sklan D., Noy Y., Hoyzman A., Rozenboim I. Decreasing weight loss in the hatchery by feeding chicks and poults in hatching trays. The Journal of Applied Poultry Research, 2000, 9: 142-148.

43. Vi e ra S.L., M or a n E.T., Jr. Effects of delayed placement and used litter on broiler yields. The Journal of Applied Poultry Research, 1999, 8: 75-81.

44. Noy Y., Gy r a A., S k la n D. The effect of early feeding on growth and small intestinal development in the posthatch poult. Poultry Sci., 2001, 80: 912-919.

45. B igot K., Mignon-Grasteau P., P i c ard M., Tesseraud S. Effects of delayed 
feed intake on body, intestine and muscle development in neonate broilers. Poultry Sci., 2003, 85: 781-788.

46. G e y ra A., Uni Z., S k la n D. The effect of fasting at different ages on growth and tissue dynamics in the small intestine of the young chick. British Journal of Nutrition, 2001, 86: 53-61.

47. Ka radas F., S u rai P.F., S park s N.H. Changes in broiler chick tissue concentrations of lipid-soluble antioxidants immediately post-hatch. Comparative Biochemistry and Physiology. Part A, Molecular \& Integrative Physiology, 2011, 160: 68-71 (doi: 10.1016/j.cbpa.2011.05.006).

48. Surai P.F., Noble R.C., S pe a ke B.K. Tissue-specific differences in antioxidant distribution and susceptibility to lipid peroxidation during development of the chick embryo. Biochimica et Biophysica Acta, 1996, 1304: 1-10.

49. S u rai P.F., F is in in V.I., Ka radas F. Antioxidant systems in chick embryo development. Part 1. Vitamin E, carotenoids and selenium. Animal Nutrition, 2016, 2: 1-11.

50. S u ra i P.F., F i s i n i n V.I. Natural antioxidants in hens' embryogenesis and antistress defense in postnatal development. Sel'skokhozyaistvennaya Biologiya [Agricultural Biology], 2013, 2: 3-18 (doi: 10.15389/agrobiology.2013.2.3eng).

51. Surai P.F., Fisini n V.I. Antioxidant-prooxidant balance in the intestine: applications in chick placement and pig weaning. J. Veter. Sci. Med., 2015, 3(1): 16.

52. A m a rase kera M., Prescott S.L., Pal me r D.J. Nutrition in early life, immunoprogramming and allergies: the role of epigenetics. Asian Pacific Journal of Allergy and Immunology, 2013, 31: 175-182.

53. Blount J.D., Metcalfe N.B., Arnold K.E., Surai P.F., Devevey G.L., Mona g h a n P. Neonatal nutrition, adult antioxidant defences and sexual attractiveness in the zebra finch. Proceedings of the Royal Society of London. Series B, Biological sciences, 2003, 270: 1691-1696.

54. Champagne F.A., R i s s m a n E.F. Behavioral epigenetics: a new frontier in the study of hormones and behavior. Hormones and Behavior, 2011, 59: 277-278 (doi: 10.1016/j.yhbeh.2011.02.011).

55. Goe rlich V.C., N at t D., E e fwing M., M a c d o nald B., J e n s e n P. Transgenerational effects of early experience on behavioral, hormonal and gene expression responses to acute stress in the precocial chicken. Hormones and Behavior, 2012, 61: 711-718 (doi: 10.1016/j.yhbeh.2012.03.006).

56. S e 11 J.L. Recent developments in vitamin E nutrition of turkeys. Animal Feed Science and Technology, 1996, 60: 229-240.

57. A p p le gat e T.J., S e 11 J.L. Effect of dietary linoleic to linolenic acid ratio and vitamin E supplementation on vitamin E status of poults. Poultry Sci., 1996, 75: 881-890.

58. Marusich W.L., Deritter E., Ogrinz E.F., Keating J., Mitrovic M., Bunn e 11 R.H. Effect of supplemental vitamin E on control of rancidity in poultry meat. Poultry Sci., 1975, 54: 831-844.

59. S oto-S la nova M.F., Se 11 J.L. Influence of supplemental dietary fat on changes in vitamin E concentration in livers of poults. Poultry Sci., 1995, 74: 201-204.

60. S oto-S a la nova M.F., Sel1 J.L. Efficacy of dietary and injected vitamin E for poults. Poultry Sci., 1996, 75: 1393-1403.

61. S oto-S a la nova M.F. Vitamin E in young turkeys: a reassessment of the requirement. In: Retrospective Theses and Dissertations. Iowa State University, 1998. Available http://lib.dr.iastate.edu/cgi/viewcontent.cgi?article $=11984 \&$ context $=$ rtd. No date.

62. M e z e s M. Effect of vitamin E treatment on early postnatal changes of vitamin $E$ status of chicken. Acta Veterinaria Hungarica, 1994, 42: 477-480.

63. G e orgi e va N.V., S toy a n chev K., B o z a k ova N., J o tova I. Combined effects of muscular dystrophy, ecological stress, and selenium on blood antioxidant status in broiler chickens. Biol. Trace Elem. Res., 2011, 142: 532-545 (doi: 10.1007/s12011-010-8782-2).

64. B a $\mathrm{ln}$ ave D., G o r m a n I. A role for sodium bicarbonate supplements for growing broilers at high temperatures. World's Poultry Science Journal, 1993, 49: 236-241.

65. Go re A.B., Qureshi M.A. Enhancement of humoral and cellular immunity by vitamin E after embryonic exposure. Poultry Sci., 1997, 76: 984-991.

66. F i s i n i n V.I., S u r a i P.F. Gut immunity in birds: facts and thinking. Sel'skokhozyaistvennaya biologiya [Agricultural Biology], 2013, 4: 3-25 (doi: 10.15389/agrobiology.2013.4.3eng).

67. Surai K.P., Surai P.F., Speake B.K., Sparks N.H.C. Antioxidant-prooxidant balance in the intestine: Food for thought. 1. Prooxidants. Nutritional Genomics and Functional Foods, 2003, 1: 51-70.

68. Surai K.P., Surai P.F., S peake B.K., S parks N.H.C. Antioxidant-prooxidant balance in the intestine: Food for thought. 2. Antioxidants. Current Topics in Neutraceutical Research, 2004, 2: 27-46.

69. M c Lean J.A., Karadas F., Surai P.F., S peake B.K., McDevitt R.M., $\mathrm{S} p$ a rks N.H.C. Lipid-soluble and water-soluble antioxidant activities of the avian intestinal mucosa at different sites along the intestinal tract. Comparative Biochemistry and Physiology, 2005, 141(B): 366-372.

70. Sura i P.F., Fot i n a A.A., F ot i n a T.I. Trudy Sumskogo natsional'nogo agrarnogo universiteta, 2012, 7: 58-61 (in Russ.).

71. Velichko O.A., Shabald in S.A., S u r a i P.F. Ptitsa i ptitseprodukty, 2013, 4: $42-45$ (in Russ.).

72. Velichko O.A., Surai P.F. Effect of an antistress composition supplied with water on 
chick growth and development. Proc. XIV European Poultry Conference. EPC, Stavanger, Norway, 2014: 551.

73. Gue rre P. Worldwide mycotoxins exposure in pig and poultry feed formulations. Toxins (Basel), 2016, 8(12): E350.

74. Murugesan G.R., Ledoux D.R., Naehrer K., Berthiller F., Applegate T.J., Grenier B., Phillips T.D., S chat z m a r G. Prevalence and effects of mycotoxins on poultry health and performance, and recent development in mycotoxin counteracting strategies. Poult. Sci., 2015, 94: 1298-1315 (doi: 10.3382/ps/pev075).

75. Es c rivá L., Font G., Ma ny e s L. In vivo toxicity studies of fusarium mycotoxins in the last decade: a review. Food Chem. Toxicol., 2015, 78: 185-206 (doi: 10.1016/j.fct.2015.02.005).

76. G hare e b K., Aw a d W.A., B ö h m J., Z e b e li Q. Impacts of the feed contaminant deoxynivalenol on the intestine of monogastric animals: poultry and swine. J. Appl. Toxicol., 2015, 35: 327-337 (doi: 10.1002/jat.3083).

77. Wu Q.H., Wang X., Y ang W., N üssle r A.K., Xiong L.Y., Kuča K., D o hnal V., $\mathrm{Z}$ h a $\mathrm{ng}$ X.J., Y u a n Z.H. Oxidative stress-mediated cytotoxicity and metabolism of T-2 toxin and deoxynivalenol in animals and humans: an update. Arch. Toxicol., 2014, 88: 1309-1326 (doi: 10.1007/s00204-014-1280-0).

78. Sorrenti V., Di Giacomo C., Acquaviva R., Barbagallo I., Bognanno M., G a lva no F. Toxicity of ochratoxin a and its modulation by antioxidants: a review. Toxins (Basel), 2013, 5: 1742-1766 (doi: 10.3390/toxins5101742).

79. Doi K., Uetsuka K. Mechanisms of mycotoxin-induced neurotoxicity through oxidative stress-associated pathways. Int. J. Mol. Sci., 2011, 12: 5213-5237 (doi: 10.3390/ijms12085213).

80. Sheu M.L., Shen C.C., Chen Y.S., Chiang C.K. Ochratoxin A induces ER stress and apoptosis in mesangial cells via a NADPH oxidase-derived reactive oxygen species-mediated calpain activation pathway. Oncotarget, 2017, 8: 19376-19388 (doi: 10.18632/oncotarget.14270).

81. Malir F., Ostry V., Pfohl-Leszkowicz A., Malir J., Toman J. Ochratoxin A: 50 years of research. Toxins (Basel), 2016, 8(7): E191 (doi: 10.3390/toxins8070191).

82. Köszegi T., Poyr M. Ochratoxin A: molecular interactions, mechanisms of toxicity and prevention at the molecular level. Toxins (Basel), 2016, 8(4): 111 (doi: 10.3390/toxins8040111).

83. Vettorazzi A, van Delft J, Lypez de Cerain A. A review on ochratoxin A transcriptomic studies. Food Chem. Toxicol., 2013, 59: 766-783 (doi: 10.1016/j.fct.2013.05.043).

84. Yang L., Yu Z., Hou J., Deng Y., Zhou Z., Zhao Z., Cui J. Toxicity and oxidative stress induced by T-2 toxin and HT-2 toxin in broilers and broiler hepatocytes. Food Chem. Toxicol., 2016, 87: 128-137 (doi: 10.1016/j.fct.2015.12.003).

85. Wu Q.H., Wang X., Yang W., Nüssler A.K., Xiong L.Y., Kuča K., Dohnal V., Zhang X.J., Yuan Z.H. Oxidative stress-mediated cytotoxicity and metabolism of T-2 toxin and deoxynivalenol in animals and humans: an update. Arch. Toxicol., 2014, 88: 1309-1326 (doi: 10.1007/s00204-014-1280-0).

86. Rezar V., Frankic T., Narat M., Levart A., S alobir J. Dose-dependent effects ofT-2 toxin on performance, lipid peroxidation, and genotoxicity in broiler chickens. Poultry Sci., 2007, 86: 1155-1160.

87. Mishra S., Dwivedi P.D., Pandey H.P., D as M. Role of oxidative stress in deoxynivalenol induced toxicity. Food Chem. Toxicol., 2014, 72: 20-29 (doi: 10.1016/j.fct.2014.06.027).

88. Li D., Ye Y., Lin S., Deng L., Fan X., Zhang Y., Deng X, Li Y, Yan H., Ma Y. Evaluation of deoxynivalenol-induced toxic effects on DF-1 cells in vitro: cell-cycle arrest, oxidative stress, and apoptosis. Environmental Toxicology and Pharmacology, 2014, 37(1): 141-149 (doi: 10.1016/j.etap.2013.11.015).

89. Osselaere A., Santos R., Hautekiet V., De Backer P., Chiers K., Ducatelle R., Croubels S. Deoxynivalenol impairs hepatic and intestinal gene expression of selected oxidative stress, tight junction and inflammation proteins in broiler chickens, but addition of an adsorbing agent shifts the effects to the distal parts of the small intestine. PLoS ONE, 2013, 8(7): e69014 (doi: 10.1371/journal.pone.0069014).

90. Wang X., Wu Q., Wan D., Liu Q., Chen D., Liu Z., Martínez-Larracaga M.R., Martínez M.A., Anadyn A., Yuan Z. Fumonisins: oxidative stress-mediated toxicity and metabolism in vivo and in vitro. Arch. Toxicol., 2016, 90: 81-101 (doi: 10.1007/s00204-0151604-8).

91. Garbetta A., Debellis L., De Girolamo A., Schena R., Visconti A., Min e rvini F. Dose-dependent lipid peroxidation induction on ex vivo intestine tracts exposed to chyme samples from fumonisins contaminated corn samples. Toxicology in Vitro, 2015, 29: 1140-1145 (doi: 10.1016/j.tiv.2015.04.018).

92. Stockmann-Juvala H., Savolainen K. A review of the toxic effects and mechanisms of action of fumonisin B1. Human \& Experimental Toxicology, 2008, 27(11): 799-809 (doi: 10.1177/0960327108099525).

93. Li u Y., Wang W. Aflatoxin B1 impairs mitochondrial functions, activates ROS generation, induces apoptosis and involves Nrf2 signal pathway in primary broiler hepatocytes. Animal 
Science Journal, 2016, 87(12): 1490-1500 (doi: 10.1111/asj.12550).

94. M a Q., Li Y., Fan Y., Zha o L., We i H., Ji C., Z hang J. Molecular mechanisms of lipoic acid protection against Aflatoxin $\mathrm{B}_{1}$-induced liver oxidative damage and inflammatory responses in broilers. Toxins (Basel), 2015, 7: 5435-5447 (doi: 10.3390/toxins7124879).

95. Shannon T.A., Ledoux D.R., Rottinghaus G.E., Shaw D.P., Dakovic A., Markovic M. The efficacy of raw and concentrated bentonite clay in reducing the toxic effects of aflatoxin in broiler chicks. Poultry Sci., 2017, 96: 1651-1658 (doi: 10.3382/ps/pew408).

96. $\mathrm{Z}$ ad e h M.H., S hahd ad i H. Nanocellulose coated with various free fatty acids can adsorb fumonisin B1, and decrease its toxicity. Colloids Surf B Biointerfaces, 2015, 134: 26-30 (doi: 10.1016/j.colsurfb.2015.06.037).

97. Nedeljković-Trailović J., Trailović S., Resanović R., Milićević D., Jova novic M., Vasiljevic M. Comparative investigation of the efficacy of three different adsorbents against OTA-induced toxicity in broiler chickens. Toxins (Basel), 2015, 7: 1174-1191.

98. Hahn I., Kunz-Vekiru E., Twarużek M., Grajewski J., Krska R., Be rth i 11 e r F. Aerobic and anaerobic in vitro testing of feed additives claiming to detoxify deoxynivalenol and zearalenone. Food Addit. Contam. Part A Chem. Anal. Control Expo. Risk Assess., 2015, 32(6): 922-933 (doi: 10.1080/19440049.2015.1023741).

99. Alp s o y L., Y a lva c M.E. Key roles of vitamins A, C, and E in aflatoxin B1-induced oxidative stress. Vitam. Horm., 2011, 86: 287-305.

100. Latypova E.N. Effektivnost' ispol'zovaniya antistressovykh preparatov «Vitaminotsid» $i$ «Medzhik Antistress Miks»v yaichnom ptitsevodstve. Avtoreferat kandidatskoi dissertatsii. [Efficiency of Vitaminocid and Magic Antistress Mix application in commercial egg production. PhD Thsis]. Orenburg, 2014. Available http://www.feedfood.com.ua/download/Latipova_PhD.pdf. No date (in Russ.).

101. Lati p ova E.N., S h a tskikh E.V., S u rai P.F. Effect of an antistress dietary supplement on the reproductive performance of layer breeders. Proc. XXV World's Poultry Congress. Beijing, China, 2016: 57.

102. Shatskikh E.V., Lat i p ova E.N., S u rai P.F. Supplying an antistress composition with water to decrease negative consequences of commercially-relevant stresses in rearing birds. Proc. XXV World's Poultry Congress. Beijing, China, 2016: 58.

103. S u ra i P.F., F is i $\mathrm{n}$ in V.I., $\mathrm{S}$ h a t s k i k h E.V., L a t y p ova E.N. Sfera: tekhnologii, kor$m a$, veterinariya, 2016, 5(2): 40-43 (in Russ.).

104. Shat skikh E.V., Lat i pova E.N., Fis in in V.I., S u rai P.F. Epigenetic effects of an antioxidant composition in layer breeder diet. Proc. XXV World's Poultry Congress. Beijing, China, 2016, p. 58.

105. F is i n in V.I., Shat sk ikh E.V., Lat y pova E.N., S u ra i P.F. Ptitsa $i$ ptitseprodukty, 2016, 1: 29-33 (in Russ.).

106. G a p o nov I.V., F ot i n a T.I., S u r a i P.F. Svinovodstvo Ukrainy, 2012, 13: 6-9 (in Russ.).

107. Bhat ta charyy A., Chat topadhyay R., Mitra S., Crowe S.E. Oxidative stress: an essential factor in the pathogenesis of gastrointestinal mucosal diseases. Physiol. Rev., 2014, 94: $329-354$.

108. L a mbert GP. Stress-induced gastrointestinal barrier dysfunction and its inflammatory effects. J. Anim. Sci., 2009, 87(14 Suppl): E101-108 (doi: 10.2527/jas.2008-1339).

109. Clark A, M a ch N. The crosstalk between the gut microbiota and mitochondria during exercise. Front. Physiol., 2017, 8: 319 (doi: 10.3389/fphys.2017.00319).

110. Gyuraszova M, Kovalcikova A, Gardlik R. Association between oxidative status and the composition of intestinal microbiota along the gastrointestinal tract. Med. Hypotheses, 2017, 103: 81-85 (doi: 10.1016/j.mehy.2017.04.011). 